\title{
Comprehensive Multi-Disciplinary Assessment Protocol for Autism Spectrum Disorder
}

\author{
Janet L. Dodd ${ }^{1, *}$, Lauren K. Franke ${ }^{2}$, Jeanette K. Grzesik ${ }^{3}$ and Jenna Stoskopf ${ }^{4}$ \\ ${ }^{1}$ Department of Communication Sciences and Disorders, Chapman University, Orange, CA, USA \\ ${ }^{2}$ Department of Extended Education, Chapman University, Orange, CA, USA \\ ${ }^{3}$ Hufstedler School of Education, Alliant International University, USA \\ ${ }^{4}$ Greater Anaheim SELPA, USA
}

\begin{abstract}
Legal mandates established under IDEA specify a student must be assessed in all areas of suspected disability. Never is this task more overwhelming than its application to the assessment of a student suspected of a diagnosis of autism. The assessment of an individual suspected of an autism spectrum disorder (ASD) diagnosis is a complex task and is dependent on the integration of information gleaned from assessments conducted by an array of professionals, each with their own distinct area of expertise. The purpose of this article is to introduce the Comprehensive Multidisciplinary Assessment Protocols-Autism Spectrum Disorder, referred to as the CMAPs, as a mechanism for organizing multidisciplinary team assessments. The CMAPs were developed in response to the challenges experienced by school-based assessment teams in developing appropriate and legally defensible assessment plans. The CMAPs provide teams a systematic, organizational, and comprehensive platform to organize the assessment of students across the spectrum of the disorder with the goal of making the task less overwhelming. Each assessment protocol considers the assessment needs of individuals with ASD using their communication skills as a preliminary starting point.
\end{abstract}

Keywords: Autism spectrum disorder, assessment, multidisciplinary.

\section{INTRODUCTION}

Legal mandates established under the Individuals with Disabilities Act (IDEA) [1] specify a student must be assessed in all areas of suspected disability. Never is this task more overwhelming than its application to the assessment of a student suspected of a diagnosis of autism. Autism is a complex disorder that impacts many facets of learning and functioning. The assessment of an individual suspected of an autism spectrum disorder (ASD) diagnosis is a complex task. An accurate identification of autism is dependent on the integration of information gleaned from assessments conducted by an array of professionals, each with their own distinct area of expertise. Assessment team members include parents, psychologist, speechlanguage pathologist, general and special education teachers, nurse/physician, and occupational therapist along with various other adjunct professionals (e.g. adaptive physical education specialist, assistive technology specialist, vision specialist, audiologist) as warranted by the individual needs of the student. School-based assessment teams are charged with the duty of coordinating the expertise of each of these team members when conducting multidisciplinary

\footnotetext{
*Address correspondence to these authors at the Department of Communication Sciences and Disorders, Chapman University, 6723 E. Espanita Street, Long Beach, CA, USA; Tel: 562-481-5475;

E-mail: dodd@chapman.edu
}

assessments. Assessments are conducted not only for the purposes of establishing eligibility, but also for guiding educational planning.

One feature compounding the complexity of this task stems from the shear spectrum of the disorder of autism. ASD encompasses a range of individuals with an even wider range of skills, abilities, and areas of need. Although there is no single battery of tests that can be applied to all students, there is consensus that an evidence-based assessment examines specific domains of functioning [2, 3]. Table 1 provides an overview of these areas and key team members generally responsible for assessing or gathering information in each area. Although assessment batteries or protocols must be individualized based on the needs of the student, there are commonalities at the various levels of the disorder. For instance, there will be some similarities in the assessment of students who present with limited to no verbal language as will there be similarities among students considered within the higher end of the spectrum.

The purpose of this article is to introduce the Comprehensive Multidisciplinary Assessment Protocols-Autism Spectrum Disorder (Supplementry A$D)$, referred to as the CMAPs, as a mechanism for organizing multidisciplinary team assessments. The CMAPs were developed in response to the challenges experienced by school-based assessment teams in 
Table 1: Areas of Assessment to Consider in Assessing a Student Suspected of an ASD Diagnosis

\begin{tabular}{|c|c|}
\hline Areas of Assessment & Responsible Person \\
\hline Autism Spectrum Disorder Assessment & Psychologist, SLP \\
\hline Executive Functioning & Psychologist \\
\hline Visual-Spatial Processing* & Psychologist, OT \\
\hline Language Functioning (syntax, morphology, semantics) & SLP \\
\hline Narrative Language Skills* & SLP \\
\hline Social Communicative Functioning & SLP, Psychologist, Teacher \\
\hline Speech Sound Production Skills & SLP \\
\hline Sensory Processing & OT \\
\hline Motor Skills & OT, PT, Adaptive PE Specialist \\
\hline $\begin{array}{c}\text { Other (vision, hearing, auditory processing, assistive technology, functic } \\
\text { assessment) }\end{array}$ & \\
\hline
\end{tabular}

Dodd [4]; *Denotes areas of assessment unique to the Atypical Communicators (AT-1. AT-2).

developing appropriate and legally defensible assessment plans. The CMAPs provide teams a systematic, organizational, and comprehensive platform to organize assessments of students across the spectrum of the disorder with the goal of making the task less overwhelming. Each assessment protocol considers the assessment needs of individuals with ASD using their communication skills as a starting point. The communication skills of students with ASD can be broadly categorized into one of three distinct communication language profiles (CLP): emergent communicator, basic communicator, and atypical communicator. Table 2 provides a detailed description of each CLP and how they compare with the American Psychiatric Association's Diagnostic and statistical manual of mental disorders (5th ed) [5] (DSM-5) ASD classification system based on severity levels (e.g. Level 3 "Requiring very substantial support"). The DSM-5, the classification and diagnostic tool of the American Psychiatric Association (APA) serves as the collective authority for psychiatric diagnosis in the United States. The DSM- 5 has substantial influence on treatment recommendations and payment for services by health care providers [5]. A CMAP was developed for each one of the CLPs, two for the Atypical Communicator-elementary (AT-1) and secondary level (AT-2), considering the unique needs of students at each of the three levels of functioning.
Students with ASD are confronted with ongoing challenges associated with communication and social interaction [5]. These students experience difficulty with social-emotional reciprocity, understanding and using non-verbal communicative behaviors, and developing and maintaining positive peer relationships [5]. Deficits in social communicative functioning (SCF) [4], as it is referred to in the CMAPs, encapsulates this intricate and active process. Deficits in SCF are one of the core features that distinguish students with autism spectrum disorders (ASD) from students with other types of disorders. The challenge is that many students with autism, particularly those on the higher end of the spectrum, often exhibit language skills (i.e. semantics, syntax and morphology) commensurate with or in some cases exceeding their age-matched peers which can camouflage true deficits when collectively examined under the broader heading of "language functioning". It is for this reason that the CMAPs examine core language skills (i.e. semantics, syntax and morphology) and skills related to SCF separately. Additionally, many students on the higher end of the spectrum perform quite well on standardized language measures but struggle considerably retelling past events or sharing experiences. Therefore, for the AT an entire section is devoted to assessing narrative language skills. The following sections will provide a brief summary of each area of assessment and considerations of functioning across the autism spectrum. 
Table 2: Description of Communication Language Profiles and DSM-5 Severity Levels of ASD

\begin{tabular}{|c|c|c|c|}
\hline \multicolumn{2}{|r|}{ CMAPs } & \multicolumn{2}{|c|}{ DSM-5 } \\
\hline Basic Communicator & $\begin{array}{l}\text { Uses symbolic forms of communication } \\
\text { (e.g. pictures, symbols, single words and } \\
\text { simple sentence patterns) for an } \\
\text { expanding range of communicative } \\
\text { purposes; communication is intentional } \\
\text { and directed toward an expanding range of } \\
\text { communicative partners }\end{array}$ & $\begin{array}{l}\text { Level 2: Requiring substantial } \\
\text { support }\end{array}$ & $\begin{array}{l}\text { Marked deficits in verbal and non- } \\
\text { verbal social communication skills; } \\
\text { limited initiation of social } \\
\text { interactions; and reduced or } \\
\text { abnormal responses to social } \\
\text { overtures from others. }\end{array}$ \\
\hline
\end{tabular}

APA [5]; Dodd [4].

\section{AREAS OF ASSESSMENT}

\section{Background}

The background information section provides a detailed description of the student's birth, developmental, and educational histories along with identifying key safety concerns of the family and any issues relating to sensory modulation. It has been the authors' experiences that many students with ASD demonstrate or have had a history of issues related to sensory sensitivities. For example, some students with autism exhibit extreme adverse reactions to noises and/or textures while others appear completely nonresponsive. In addition, it is also important to document any medical interventions such as prescriptive medications, special diets, and non-customary therapies which may have been implemented. A thorough description of a student's background provides critical information necessary for an accurate diagnosis and for effective intervention planning to occur.

\section{Observations}

Direct observation (DO), observing the student within the context of their naturalistic environment (e.g. classroom, playground/recess, daycare/after-school program and home environment), provides the opportunity to document "environmental contingencies" [6] that seem to influence the occurrence of certain behaviors. DO provides insight regarding the student's language and communication skills and the impact of these skills on his/her interactions with others. Valuable information gleaned from observations assists with both the process of customizing the CMAPs as well as providing descriptive data relevant to the student's overall awareness, responsiveness and interaction with age-matched peers and familiar adults. A study by Duchan [7] found that "the way someone with autism is regarded and described is strongly related to what the describer wants to accomplish". It is for this reason that observations need to be conducted by multiple observers with varying professional backgrounds and relationships to the student in settings that are both structured and unstructured. As Wilkinson [8] stated, "Direct observation of the student in both structured and unstructured settings improves accuracy in the identification of ASD". Table 3 provides a sample list of considerations by CLP when conducting an observation of a student:

\section{Autism Spectrum Disorder Assessment}

The purpose of this area of assessment is to provide recommendations for best practices for schoolbased evaluation teams when selecting an assessment 
Table 3: Observational Considerations Across the CLPs

\begin{tabular}{|c|c|c|}
\hline Emerging Communicator & Basic Communicator & Atypical Communicator \\
\hline \hline Awareness of peers & Range of communicative partners & Responsiveness to peers \\
\hline Ability to follow peer models & Reciprocity of interactions & Initiation strategies \\
\hline Ability to follow familiar routines & $\begin{array}{c}\text { Interpretation and awareness of the } \\
\text { communicative needs of partners }\end{array}$ & \\
\hline Rate of initiation and responsiveness & Types of interactions & \\
\hline Ability to establish and maintain joint attention & Responsiveness to peers & \\
\hline
\end{tabular}

tool for the assessment of ASD symptomatology. The assessment for a diagnosis of an autism spectrum disorder using the DSM- 5 is much more specific and focuses on the discrete symptoms and characteristics of ASD. In contrast, a school eligibility criterion (e.g. IDEA) addresses specifically the impact of general symptoms on access to curriculum. Because of this, it is important for teams to evaluate for diagnostic criteria and the impact of symptoms on the student's daily functioning. Use of an assessment tool specific to autism will help teams determine subtle differences between ASD eligibility and other categories such as Emotional Disturbance and Intellectual Disability.

\section{Cognitive Functioning}

Cognition is the mental action or process of acquiring knowledge and understanding through thought, experience, and the senses [9]. The assessment of cognitive functioning typically consists of the administration and interpretation of a cognitive, or intelligence test. While cognitive functioning is not considered to be a core deficit in ASD, cognitive assessment is considered to be a core component of a comprehensive evaluation. Test results combined with adaptive skills and other assessment information indicates whether the student has co-occurring intellectual delays (ID).

Due to the unique developmental and behavioral issues associated with assessing students with ASD, selection of the particular IQ test requires careful consideration and the evaluator should have experience working with students with ASD. There is no "best test" for measuring intellectual functioning in persons with ASD [10]. Klinger, O'Kelley and Mussey
[10] stress that determining the student's level of expressive and receptive language is essential when choosing an appropriate measure to obtain valid results. There are cognitive tests that measure nonverbal skills only, while others measure both verbal and non-verbal skills. A non-verbal student would not have the expressive language skills necessary to respond to the prompts on a verbal test. Given receptive skills that would enable the non-verbal student to respond to verbal prompts by pointing to a picture or matching blocks to a prompt design, a non-verbal cognitive test could provide valuable insight regarding the student's basic problem-solving skills. However, skills demonstrated in a test situation may not generalize to real-life problem-solving situations and may lead assessment teams to conclude that the student has global intact and average non-verbal problem-solving or cognitive skills. Results of any cognitive skills tests need to be carefully interpreted within the context of results of other assessments in the areas of adaptive skills, executive skills and language skills.

Use of the CMAPs provides the IEP team guidance in selecting an appropriate IQ test. There is no single cognitive profile that specifies ASD eligibility; however, beginning in early elementary school students with ASD will show various patterns of cognitive functioning across the CLP profiles. Knowledge of cognitive functioning will contribute valuable information about a student's educational needs and potential outcome that needs to be considered during the assessment process. Although some students through the kindergarten age range may show an increase in cognitive functioning it is expected from childhood through adulthood scores of intellectual functioning in individuals with autism remain relatively stable [11-13]. 
In summary, it is important to recognize that the profile of cognitive strengths and weaknesses of students with autism tend to be uneven. It is the identification of the students' strengths and weaknesses on cognitive measures that are of importance and not their overall score. Approximately $10 \%$ of children with autism show unusual areas of ability or splinter skills [14]. These skills could be considered personal strengths but they may not be indicative of performance in other areas or related skills. For example, a 7-year-old student who spells and reads words beyond his or her grade level but cannot understand what they read would be such an example. Unfortunately the student does not demonstrate functional use of this skill. Some students with autism perform better on non-verbal measures. Some students with higher or at-average non-verbal intelligence $(>80)$ may fail to develop adequate oral language skills [15]. In this situation non-verbal IQ scores can be misleading [16].

\section{Executive Functioning}

Executive functions are often identified as the "boss" of the brain. "The executive functions consist of those capacities that enable a person to engage successfully in independent, purposive, self-serving behavior" [17]. Our ability to maintain flexibility and adapt to changing situations in what seems to be an effortless and fluid process is a direct result of executive functions which enable us to plan, initiate, monitor, evaluate, modify, and change [18]. An individual with impaired executive functioning may experience difficulty working independently, performing self-care activities or maintaining normal social relationships in spite of average intelligence. Initiation and working memory along with other aspects of executive functioning are related to the deficits in adaptive skills that are often exhibited by high functioning students with ASD [19]. Flexibility, organizational skills, planning and self-monitoring are executive functioning skills typically impaired in children with ASD [20].

Executive dysfunctions (EDF) are often reported in ASD but are not unique to ASD. Students with attention deficit disorder demonstrate delays in all areas of executive skills. Executive dysfunction is not a core deficit that leads to the specific issues in ASD. Rather EDF is secondary to other earlier appearing deficits [20]. Since EDF is often reported in ASD, it is an area that requires assessment to determine how deficits may be impacting the student's access to the curriculum and progress at school. Maladaptive patterns of behavior in the classroom can be directly related to EDF. Knowledge of a student's EF strengths and weaknesses along with overall learning strengths and weaknesses can assist IEP teams in developing appropriate programs for students with ASD.

Executive functioning skills are linked with the frontal lobe, one of the last parts of the brain to mature; therefore, initial executive functioning assessment is not generally recommended until the child is at least five years of age [21]. Executive skills are not formally assessed using the CMAPs until the child is considered an atypical communicator. The atypical communicator may have the skills required to respond to the task demands of more formal tests of executive functioning. In addition, the responses on one of the observational rating inventories of executive functioning, such as the BRIEF, may reveal a pattern of strengths and weaknesses for a student at this level.

\section{Visual Spatial Processing}

The assessment of visuospatial functions includes evaluation of perceptual skills, constructional skills, and spatial awareness [22]. A student with deficits in this area may write unevenly, draw poorly, complete puzzles poorly, bump into things, cover one eye while writing or reading and concentrate on parts of a task rather than the whole. According to Jiron [21], "The visuospatial domain includes the receptive visual abilities to distinguish color, shape, distance, details and overall gestalt. It also refers to the expressive abilities involving eye-hand coordination, such as scanning, tracking, and copying; grasping or catching a targeted item; and drawing or building a replica of a design or object". Visuospatial skills and language skills are essential for a student to have academic success since much of what is learned in school has a language or visuospatial basis [23]. Students with autism often perform in the average to above average range in this area, indicating that visuospatial functioning is frequently an area of relative strength [23].

\section{Memory}

Terms related to memory are classified in a number of ways [24]. Explicit or declarative memory refers to a conscious awareness of recall. It includes episodic memory, which is memory for the singular events that a person recalls; and semantic memory, which is knowledge about the world that is not autobiographical [25]. Assessments that are useful in evaluating episodic memory include list-learning, story-learning, 
and design-learning tests. The results of these assessments help evaluate encoding, or learning of novel information, and consolidation or retention of information [24]. Assessments that are useful for measuring semantic memory skills include tests of word fluency or category fluency, vocabulary, naming and free association. The results of these assessments help to evaluate the student's ability to organize information in order to remember it later [24].

Implicit or procedural memory is an unconscious form of memory such as how to ride a bicycle or how to tie your shoes. The individual is not aware of this system or that they are using implicit memory. Implicit or procedural memory is often an area of strength for students with autism [26].

Short-term memory is the ability to hold information for a short period of time and then to encode and retain this information. It is assessed using tests such as digit span forward, paragraph passage recall and tests measuring recall of visual designs. Long-term memory refers to the ability to consolidate and store information in order to retrieve it at a later time or after a competing stimulus has been introduced [24].

Minshew and Goldstein [27] point out that memory and learning functions are selectively impaired in children with autism spectrum disorder. In fact, much of the dysfunction in memory is related to failure to utilize organizational strategies in both visual and auditory modalities. In addition, they discovered that memory performance decreases as the complexity of the information to be remembered increases.

Working memory is a limited capacity system that provides temporary storage in order to manipulate information for complex cognitive tasks such as learning and reasoning. Baddeley [28] coined terms related to working memory's two parts. One part is the phonological loop, which is temporary storage for speech-based and acoustic information. The visuospatial sketchpad is for the manipulation of visualspatial information. One study of working memory found no deficit in verbal working memory, or in the use of the phonological loop, in high functioning children, adolescents and adults with autism. It did, however, discover that the people in the study with autism did more poorly than controls on the spatial working memory tasks, or visuospatial sketchpad activities [29].

Memory skills are not formally assessed using the CMAPs protocol until the student is considered an atypical communicator. The atypical communicator may have the skills required to respond to the task demands of more formal tests of memory functioning. Students who are basic or emerging communicators would have difficulty responding to the prompts in a formal test of memory functioning; however, the procedural memory skills of the emerging communicator can be observed as they demonstrate learned routines such as tying their shoes or riding a bicycle.

\section{Language Functioning}

Difficulties with language and communication are one of the hallmark characteristics of students with autism and there is wide variability across the spectrum of the disorder [30]. Students range from being nonverbal to demonstrating above average language skills. While not all students on the spectrum will have language deficits related to formulating sentences, by definition all will have difficulty with aspects of social communication or the pragmatic aspects of language. Receptive language skills can vary from very limited understanding of language to subtle deficits that are often missed due to average to above average scores on formal language tests. Examples include understanding idioms, jokes, sarcasm and knowing what to say in conversations. In fact it is not uncommon to be blinded by the strengths students with higher functioning variations of ASD display. Due to the wide variability of skills and the subtlety of the deficits for some students it is critical that all students suspected of being on the spectrum be provided with a thorough language assessment.

There are two purposes for assessment in this area. The first is to clarify the student's pattern of language strengths and weakness and how this pattern impacts the student's access to the curriculum. The development of language and communication skills is important for all students with ASD and can impact school success. It is critical that SLPS be knowledgeable in common core state standards and the critical role language plays in achieving those standards at each grade level. The second purpose is to determine if the student has primarily a language delay or disorder or if the two are co-occurring. Table 4 provides a list of language considerations across the CLPs.

\section{Narrative Language Skills}

Narrative language is an important aspect of life. People use narratives to share their experiences, connect with others, and make sense of the world. 
Table 4: Language Considerations Across the CLPs

\begin{tabular}{|c|c|c|}
\hline Emerging Communicator & Basic Communicator & Atypical Communicator \\
\hline $\begin{array}{l}\text { Discrepancy between language skills and } \\
\text { other areas of functioning (e.g. gross motor) }\end{array}$ & $\begin{array}{l}\text { Uses echolalia for an expanding range of } \\
\text { purpose. }\end{array}$ & $\begin{array}{l}\text { May show no delays in language and/or } \\
\text { exhibit peculiar language patterns }\end{array}$ \\
\hline $\begin{array}{l}\text { Less responsive to someone calling their } \\
\text { name to not responding at all }\end{array}$ & $\begin{array}{l}\text { Receptive language skills are often weaker } \\
\text { than expressive language skills }\end{array}$ & $\begin{array}{l}\text { Strong lexical knowledge but may also } \\
\text { exhibit abnormal use such as metaphorical } \\
\text { language (i.e. modification of root words to } \\
\text { an oddly sounding but discernible version of } \\
\text { the target word). }\end{array}$ \\
\hline Limited to no expressive language & $\begin{array}{l}\text { Difficulty with deixis or the use of language to } \\
\text { indicate shifting reference (e.g. referencing } \\
\text { "you" vs. "l") }\end{array}$ & $\begin{array}{l}\text { Use and understanding of mental state } \\
\text { terms (e.g. thinking, knowing, emotional } \\
\text { states) may be absent or used } \\
\text { inappropriately }\end{array}$ \\
\hline $\begin{array}{l}\text { Difficulty understanding labels of common } \\
\text { objects or responding to simple routine } \\
\text { commands }\end{array}$ & $\begin{array}{l}\text { Grammatical deficits consistent with specific } \\
\text { language impairment. }\end{array}$ & $\begin{array}{l}\text { May use pedantic or overly formal } \\
\text { professor-like speech }\end{array}$ \\
\hline
\end{tabular}

Adapted from Tager-Flusberg, Paul, \& Lord [31].

Narrative assessment is a critical component of the CMAPs protocol. It is deemed important not only because it is a key life skill, but also because it may be a more authentic assessment of language skills. Some students with ASD perform adequately on static measures of language and their problems are better identified on tasks that rely on processing dynamic complex information that is more typical of everyday interactions. Narrative skills showcase higher-level language and cognitive skills and are a predictor of future literacy and social skills. There are several different types of narratives:

- Scripts: expressing knowledge of familiar, recurring events.

- Recounts: retelling a personal experience when prompted and are shared by listeners.

- Event casts: explaining an ongoing activity, reporting on a factual scene, or telling about a future plan.

- Accounts: explaining personal experience without the prompts and are not shared by the listener.

- $\quad$ Fictional story retelling: the child retells a story that he/she read or which was read to him/her.

- $\quad$ Fictional story telling: the child creates and tells a story from visual stimuli such as sequence pictures or a single picture.
Accounts of personal experiences and retelling fictional stories are often used to assess narrative skills [41, 42, 44]. Narrative assessment entails collecting and analyzing narrative language samples. Stories are examined at two levels: the micro and macro levels. The micro-level assessment examines the grammatical and semantic elements of the narrative whereas the macro-level assessment examines the organization and structural elements of the narrative. Contrastively, conversational language samples examine the back and forth exchange between conversational partners. In their narratives, students with ASD:

- Use less complex sentences [32] but some exhibit advanced language skills [33].

- $\quad$ Make more syntactic errors [32].

- Focus on insignificant details.

- Produce narratives that are significantly less coherent.

- $\quad$ Fail to use the "gist" of the story to organize their narratives coherently [34].

- Experience difficulty putting story retellings together as a meaningful chain of events [35].

- Fail to weigh events with regard to their importance [36].

- $\quad$ Tend to have incomplete episodes [37]. 
- $\quad$ Produce stories that are not well organized [37].

- $\quad$ Fail to link important events coherently in their retellings [37].

- Include information that was bizarre, inappropriate or irrelevant [38].

The narrative assessment is typically completed by the SLP and a variety of standardized, nonstandardized and dynamic assessments are available. Standardized measures depending on the student's age include Test of Narrative Language (TNL) [39], Narrative Assessment Protocol (NAP) [40] and the Narrative Language Measures (NLM) [41]. The second two measures are for students ages 3-6 and are available online at no cost. The Systematic Analysis of Language Transcripts (SALT) [42] software also has a narrative database. Informal assessment can be conducted using guidelines from Hedberg and Westby [43], "Analyzing Storytelling Skills". McCabe and Rollins [44] provide guidelines for obtaining and analyzing personal narratives.

Due the importance of narratives as well as to the wealth of information that can be collected, narrative assessment is a valuable component of the CMAPs protocol.

\section{Social Communicative Functioning}

Persistent challenges in the areas of social interaction and communication (verbal and non-verbal communicative behaviors), collectively referred to as social communicative functioning in the CMAPs, are a core cognitive deficit of students across the spectrum of the disorder of autism [5]. In spite of some students with ASD having average to above average language skills, which is often characteristic of children with high functioning autism and Asperger's syndrome, social communication remains an area of considerable challenge which hinders their ability to develop positive peer relationships and accessibility to the curriculum. Social communication encapsulates our ability to exchange meaningful messages which is contingent on our own adaptability (e.g. modifying our verbal and non-verbal communicative behavior so others develop positive thoughts about us) to the situation and those involved [45]. Ability to adapt our behavior based on the situation and students who have deficits in this area but who do not demonstrate repetitive behaviors will receive a diagnosis of Social Communication Disorder rather than ASD under the revised DSM-5, whereas in the school setting, an eligibility of autism or speechlanguage impairment would be determined based on the student's profile.

The CMAPs model of assessing SCF was influenced by Coggins, Olswang, Carmichael Olsson, \& Timler's [46] model of social communicative competence (SCC). "According to Coggins and colleagues, SCC is dependent on the integration of language, social cognition, and higher order executive skills. An individual's social communicative behaviors are a direct reflection of this integration [4]". SCF is a multifaceted process that requires the integration of social cognition (i.e. joint attention, emotion recognition, theory of mind), pragmatics (e.g. conversational skills), and social problem abilities, all of which are executed and recognized in one's social communicative skills (i.e. play skills, social skills, and friendship skills). Figure 1 provides a schematic of this intrinsic process that is yet so complicated for children with ASD.

Social Cognition is the broad term used to encapsulate the processes which we use to encode process, and use information in social contexts enabling us to make sense of the behaviors of others [47]. It is dependent on our ability to establish and maintain joint attention; recognize, interpret, and respond appropriately to not only others' emotions but our own as well; and our recognition that others' perspectives are influenced by the knowledge they possess. Social cognition follows a developmental sequence of acquisition with earlier developing skills being precursors to later developing skills. In fact, joint attention is predictive of later social cognitive development [48].

Pragmatics refers to specific behaviors used to convey messages [49] and involves language skills used in real conversational contexts [50]. It has been defined as "the range of communicative functions (reason for talking), the frequency of communication, discourse skills (turn taking, topic maintenance and change), and flexibility to modify speech for different listeners and social situations" [50]. There are a variety of observational tools and non-standardized protocols for eliciting pragmatic behaviors (e.g. Pragmatic Protocol [51], Communication Checklist-2 [52], Pragmatics Profile from the CELF-5 [53], Peanut Butter Protocol [54]). Both types of protocols/tools examine a language sample in naturalistic interactions. Examples of key areas that are noted include Sustaining conversation (e.g., turn taking, topic selection) and repairing communication breakdowns. 


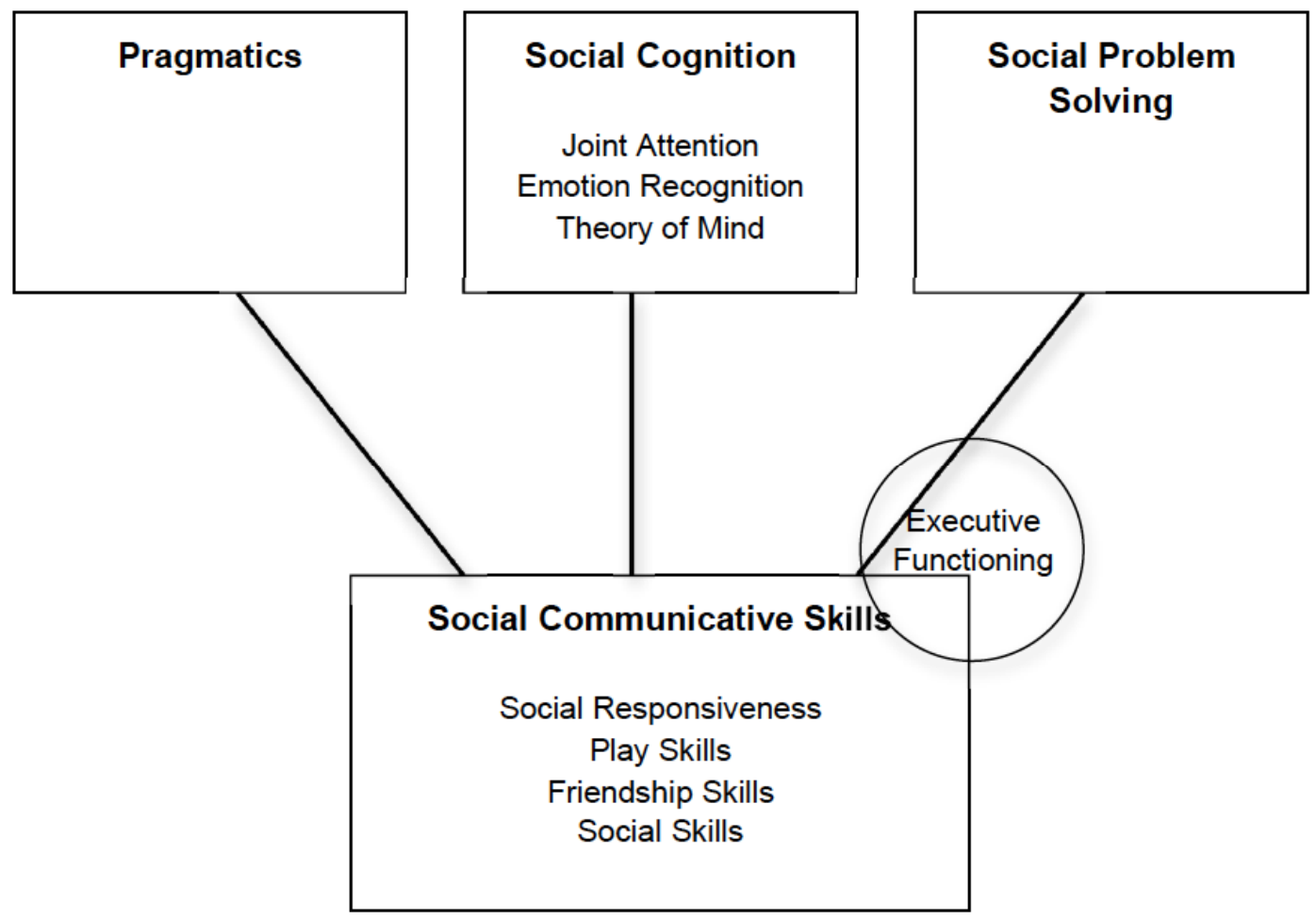

Figure 1: Social Communicative Functioning.

Social problem solving comprises the self-directed and conscious cognitive-behavioral process that enables us to respond to everyday naturally occurring problems [55]. This intuitive process allows us to recognize a problem in the moment, making immediate adjustments to rectify the situation.

To fully understand the student's breakdown in functioning it is necessary to integrate information assembled from observations and probes with the results of standardized measures (e.g. Social Language Development Test -Elementary (SLDT-E) [56], Test of Pragmatic Language-2 (TOPL-2) [57]). Students with high forms of autism (e.g. HFA, AS) may not show deficits on standardized tests due their static nature and many items can be answered correctly based on information the student can recall about a particular social situation. Correct responses do not necessarily indicate application of this knowledge.

Social communication functioning is a complex area to fully understand and assess. Evaluators will want to keep in mind the interactions of the various components as it will guide treatment planning.

\section{Speech Sound Production Skills}

Speech, our ability to formulate sounds into words, not only impacts our ability to create intelligible and understandable utterances but influences the perceptions of ourselves by others. Speech sound production skills can be better understood if we consider them in terms of segmental and suprasegmental aspects. Deficits in the segmental aspects of speech are collectively referred to as speech sound disorders (SSDs) $[58,59]$ and include articulation and phonological disorders along with disorders such as Childhood Apraxia of Speech (CAS). A student who is identified as having an articulation disorder has difficulty with the motoric execution of sound production resulting in aberrant or deviant speech (e.g. lateralized /s/) [60, 61]. A child who is demonstrating a phonological disorder is having difficulty using sounds contrastively to distinguish meaning. For example a child who is substituting "t" for " $k$ " will say "tea" and "key" as "tea". Childhood apraxia of speech (CAS) is a neurological childhood (pediatric) speech sound disorder in which the precision and consistency of movements underlying speech are impaired in the absence of neuromuscular deficits (e.g. abnormal reflexes, abnormal tone). CAS may occur as a result of known neurological impairment, in association with complex neurobehavioral disorders of known or unknown origin, or as an idiopathic neurogenic speech sound disorder [62]. Speech sound disorders vary in degree of severity taking into consideration the age of the student and impact on the student's ability to be understood by peers and familiar adults. 
Supra-segmental aspects, on the other hand, refer to how we modulate the meaning of our speech through prosody and voice [63]. Supra-segmental aspects of speech can be categorized into three broad categories: grammatical prosody, pragmatic prosody and affective prosody [63]. Grammatical prosody refers to how we use stress and pitch to denote meaning of a word or sentence such as using rising intonation at the end of a sentence to indicate a question or using pauses to influence the intended meaning of a sentence. For example, the intended message of the sentence, "Trevor, the coach is here" changes when we added an additional pause after "coach" and the sentence becomes, "Trevor, the coach, is here". Pragmatic prosody is illustrated in how we use stress to draw our listener's attention to, or to highlight a particular aspect of a sentence or conversation. Affective prosody refers to our individual speech style and how we modulate our speech dependent on the social situation. For instance, we may use a more formal speech style when conversing with a person of authority such as an employer or professor compared to engaging in a conversation with a close friend. Additionally, our emotional state influences the suprasegmental aspects of our speech and the urgency of our statements.

Collectively, segmental and supra-segmental aspects of speech influence not only how we are understood by others but how we are perceived.

\section{Developmental/Pre-academics/Academics}

"Assessment of academic ability, even in younger children, is helpful for the purposes of educational decision making" [3]. Some students with autism spectrum disorder often have good rote memories and an ability to amass great quantities of facts and will often perform well on typical tests of educational achievement in the elementary school years. However, most often, they have a limited ability to apply the acquired knowledge into functional skills and to generalize their skills across environments and tasks [64]. The most consistent area of weakness in academic skills for students with autism is reading comprehension, which also results in a weakness in the area of math applications. Areas of strength often include decoding words and learning facts such as multiplication tables. It is important that assessment include areas of academic strengths as well as academic weaknesses so that data-based educational recommendations can be made and academic progress monitored.

\section{Adaptive Functioning}

One of the key domains in a core autism assessment is adaptive skills. Measuring adaptive skills is important for setting appropriate goals and is required if a diagnosis of intellectual disability is being considered. Essentially, adaptive skills assessments measure real-life functioning across environments via rating scales completed by interview of parent/caregiver and teacher. "Adaptive abilities largely determine whether an individual requires constant supervision or is capable of some independence" [3]. Many students with autism demonstrate adaptive behavior levels that are lower than their intellectual ability as measured on standardized tests. The domains of functioning measured by adaptive scales are communication, daily living skills, socialization and for younger children, motor skills.

Current studies comparing intelligence, severity of autism characteristics and adaptive skill levels indicate that individuals with autism "do not use their cognitive abilities appropriately in the service of improving their adaptive skills, especially their social skills" [65]. As students become older, the gap between their intelligence and their adaptive skills appears to become wider, indicating that they are failing to acquire adaptive skills that are comparable to their cognitive skills and their age. They are not increasing their adaptive skills in order to become independent adults, capable of self-management in order to live independently and to hold down a job. The importance of direct instruction and progress monitoring of the functional skills of communication, daily living and socialization must be recognized as we prepare our students with autism to live more independently once they leave the school system.

The most widely used measure of adaptive skills with students with autism is the Vineland Adaptive Behavior Scales, $2^{\text {nd }}$ Edition (Vineland ${ }^{\mathrm{TM}}-$ II) [66]. These scales can be used with the physical, functional, basic, and atypical communicators to measure adaptive skills.

\section{Behavior/Self-Regulation/Emotional Functioning}

A structured approach to the assessment of behavior is critical for students with autism. In addition to standardized instruments such as the Child Behavior Checklist (CBCL) [67, 68], a series of behavioral observations are critical [69]. Specific attention should be given to the student's behavior in a variety of 
environments with data collected on behavioral skills such as emotional self-regulation, attention, and compliance to adult requests within the context of the student's developmental level, social skills, and language and communication skills. Interviews of parents, caregivers, teachers and school staff will provide valuable insight into the function of the student's behavior. Developing a hypothesis of the function of behaviors will provide the basis for a functional behavioral assessment. Accurate recording of baselines for various behaviors will allow the educational team to chart growth in the student's repertoire of positive behaviors as interventions are systematically applied.

In addition to intervention planning, behavioral assessment may help to identify underlying issues related to anxiety, depression, or other internalizing mental health issues. Anxiety for a student with autism may manifest itself in rigid inflexibility, intense intolerance of stress [70] or disruptive behavior that results in escape from fearful situations. Externalizing problems such as hyperactivity, impulsivity, anger and aggression, may be identified in a behavioral assessment as well. These underlying, co-morbid mental health issues may exacerbate difficult behaviors and require unique behavioral and therapeutic supports to enable the student with autism to make educational progress in the least restrictive environment.

Systematic observation of the behavior of the physical, functional, basic and atypical communicators across environments will provide information to form a baseline prior to intervention as well as data that is critical to the development of interventions, programs and strategies to address behavior. Interview of parent, caregiver and teacher as well as standardized assessment will support the team in creating targeted interventions for all CMAPs communicators.

\section{Sensory Processing}

Sensory processing skills are defined as neurobiological processes by which the central nervous system registers sensory input [71-73]. Sensory input comes from multiple senses which include tactile (touch), olfactory (smell), proprioceptive (joint \& muscle), vestibular (movement) and auditory (hearing) [71]. Feedback from these senses enables a person to recognize, assimilate and organize everyday sensory information in order to interact effectively within an environment, which creates the foundation for learning [71]. Dunn's [72] model of sensory processing expands on this line of thinking, to include how the processing of sensory information is varied between individuals, as individuals have varying levels of thresholds which may impact behavior.

Multiple researchers and authors have reported that children with ASD demonstrate difficulty with skills related to sensory integration. According to Dunn [73], "people who have autism spectrum disorders (ASD) have more intense reactions than their peers, which may be one of the contributing factors to their differences in responding in particular situations". Students with ASD are more likely to show elevated scores on sensory questionnaires, indicating sensitivities, in the areas of under responsive/seeks sensation, auditory filtering, tactile sensitivity and taste and smell sensitivity [74]. An additional study indicated that children diagnosed with ASD scored significantly different on questionnaires measuring sensory processing skills in comparison to children with no diagnosis [75]. Anzalone and Williamson [76] note, "many children with autism have problems in modulating their response to sensory input, and maintaining optimal arousal and focused attention". Sensory processing is often an area of concern that has impact on other domains of functioning, including play and social activities [77].

\section{Motor Functioning}

For the purposes of the CMAPs, motor skills encompass fine and gross motor skills along with visual motor skills necessary for successful execution of fine and gross motor skills. Fine motor skills are defined as the motor skills used by the hands and fingers. In an educational setting, a student utilizes fine motor skills to participate in a variety of activities such as handling classroom tools (e.g. scissors, crayons, and markers), opening containers at mealtime, opening and closing their back pack, putting on and taking off clothing items (e.g. zippers, buttons) and maintaining grasp on play equipment. Gross motor skills are defined as skills involving arms, legs and core. A student who has gross motor deficits often has an awkward gait and appears clumsy. An adapted physical education teacher may assess the student's skills in running, jumping, hopping, and kicking a ball. These skills are typical playground and recess skills that will impact the student's ability to access outside play opportunities with peers.

Visual motor skills refer to the ability to coordinate vision with the movements of the body. Vision is 
involved in all of our movements whether they are gross motor or fine motor. At school, visual motor skills are needed for participation in playground ball activities, and paper and pencil tasks such as folding paper, tracing and cutting on a line, copying and drawing letters, writing numbers and shapes. Visual perception and visual tracking skills are additional visual skills that may be assessed. Visual perception is the cognitive process of obtaining and interpreting visual information. It includes skills of spatial relationships, visual discrimination, figure-ground, visual closure, and visual memory. An assessment conducted by an occupational therapist would examine fine motor and visual motor skills. Additional testing in these areas may also be completed by the school psychologist in the visual processing assessment. Gross motor skills can be assessed by an OT; however they are often evaluated by a professional in adapted physical education or a physical therapist.

Children with a diagnosis of ASD often demonstrate difficulty with motor tasks [78]. One study suggests that children with autism demonstrate impaired coordination skills in comparison to typically developing children when measured on a standardized motor test [79]. An additional study indicated similar findings, noting that children with autism demonstrated poorer strength, and agility motor skills [80].

\section{CLINICAL IMPLICATIONS}

Although there is no specific "recipe" for a thorough assessment of a student who is suspected of having autism, there is a structure within which assessment teams can operate. The CMAPs provide a systematic, organized structure based on the individual student's level of communication skills to support teams in developing plans for thorough and legally defensible assessments. Legally defensible assessments comply with federal mandates which stipulate students must be assessed in all areas of suspected disability by high quality professionals [1]. Each section of the structure requires assessment utilizing research-based measures and best practice skills of the professionals involved.

What makes the CMAPs unique from other ASD assessment protocols is that they were developed based on a multidisciplinary model to individualize the assessment needs of a student based on a preliminary estimate of their language and communication needs. The CMAPs take into consideration all areas of potential weakness a student with ASD may exhibit at each level of functioning. Another aspect that makes the CMAPs different from other protocols is the unique and detailed manner in which language and communication skills are examined with respect to language functioning, narrative language skills, and social communicative functioning. This allows assessment teams to tease out strengths and weaknesses that impact the student's ability to develop positive peer relationships and access the curriculum. With this data gathered, the team can develop goals that reasonably match the student's predicted pace of achievement and development. Although intended to be used by school district teams the CMAPs can also be used by parents to assist them in understanding the assessment needs of their child.

Each member of the team must consider the student's cultural and linguistic background when choosing and interpreting assessments. It is critical to distinguish between characteristics of autism and characteristics related to cultural differences. Behaviors that may be viewed as autism symptomatology in one culture may have an interpretation that is markedly different in another culture [58]. For example, failure to establish and maintain eye contact is often considered a characteristic of autism however in some cultures direct eye contact can be viewed as disrespectful or rude [81]. The assessment team will need to become familiar with the behaviors that may be demonstrated by the student that are unique to their cultural background and not symptoms of autism.

In practice each member of the assessment team chooses assessments that will provide the most accurate information regarding the student's present levels of functioning for eligibility determination and educational planning purposes, another important conclusion of assessment. Working as a team, the professionals involved in the assessment should consider the observations and performance of the student across disciplines and environments. Assessment reports should not be viewed as individual team members providing separate reports that are simply cut and pasted into an overall document but rather a gathering of data to contribute to a larger and more meaningful conclusion. The authors have found it extremely beneficial to meet regularly as a team to engage in professional dialogue regarding a student's performance on specific tests and to discuss their observations of the student's skills in different environments. This provides an opportunity for team members to share results, identify consistencies, and 
discuss potential reasons for discrepancies in the assessment findings. For example, each member of the team might have data to provide regarding the student's gross motor skills through their own observations of the student throughout their day(e.g. how the student navigates his or her way around desks, chairs and tables). Memory is another area of functioning that is often evaluated and observed across disciplines. It is customary to assess memory as part of the battery of tests in the areas of cognition and executive functioning typically administered by the psychologist. Additionally, memory is also observed in various measures of language functioning administered by the speech-language pathologist. The team discussion should review the various test results and observations of the student across disciplines as strengths and weaknesses are discussed. Discrepancies and consistencies in scores may lead to valuable information regarding how the student functions leading to a more accurate diagnosis and more specific interventions. These professional discussions lead to an assessment report that addresses strengths and weaknesses across disciplines, rather than a series of individual reports, in order to more accurately diagnose and address the student's educational needs. The CMAPs is a tool to guide teams through the assessment process of a student suspected of an ASD diagnosis for the purposes of establishing eligibility and developing a comprehensive and specific intervention program that will address the student's unique educational needs.

\section{SUPPLEMENTARY DATA}

The supplemental data can be downloaded from the journal website along with the article.

\section{REFERENCES}

[1] Individuals with disabilities education act (2004). 20 U.S.C. $\S 1400$

[2] Filipek PA, Accardo PJ, Baranek GT, et al. The screening and diagnosis of autism spectrum disorders. J Autism Dev Disord 1999; 29: 439-84. http://dx.doi.org/10.1023/A:1021943802493

[3] Ozonoff S, Goodlin-Jones BL, Solomon, M. Evidence-based assessment of autism spectrum disorders in children and adolescents. J Clin Child Adolesc Psychol 2005; 34: 523-40. http://dx.doi.org/10.1207/s15374424jccp3403 8

[4] Dodd J. Thinking outside of the assessment box: assessing social communicative functioning in students with ASD. Perspect Sch Based Iss 2010; 11: 88-98. http://dx.doi.org/10.1044/sbi11.3.88

[5] American Psychiatric Association. Diagnostic and statistical manual of mental disorders. $5^{\text {th }}$ ed. Arlington (VA): American Psychiatric Publishing; 2013.
[6] Ramsey MC, Reynolds CR, Kamphaus RW. Essentials of behavioral assessment. New York: Wiley and Sons; 2002.

[7] Duchan JF. Describing the unusual behaviors of children with autism. J Commun Disord 1998; 31: 93-112. http://dx.doi.org/10.1016/S0021-9924(97)00084-1

[8] Wilkinson LA. Autism and asperger syndrome in schools. Philadelphia (PA): Jessica Kingsley Publishers; 2010.

[9] Stevenson A, Lindberg CA. New oxford american dictionary. $3^{\text {rd }}$ ed. New York: Oxford University Press; 2010. http://dx.doi.org/10.1093/acref/9780195392883.001.0001

[10] Klinger LG, O'Kelley SE, Mussey JL. Assessment of intellectual functioning in autism spectrum disorders. In: Goldstein S, Naglieri JA, Ozonoff S, editors. Assessment of autism spectrum disorders. New York: Guilford; 2009. p. 20952.

[11] Nordin V, Gillberg C. The long-term course of autistic disorders: update on follow-up studies. Acta Psychiatr Scand 1998; 97: 99-108.

http://dx.doi.org/10.1111/j.1600-0447.1998.tb09970.x

[12] Sigman M, McGovern CW. Improvement in cognitive and language skills from preschool to adolescence in autism. $J$ Autism Dev Disord 2005; 35: 15-23. http://dx.doi.org/10.1007/s10803-004-1027-5

[13] Shea B, Mesibov G. Age-related issues in the assessment of autism spectrum disorders. In: Goldstein S, Naglieri JA, Ozonoff S, editors. Assessment of autism spectrum disorders. New York: Guilford; 2009. p. 117-37.

[14] Lord C, McGee JP. Educating children with autism. Washington DC: National Academy Press; 2001.

[15] Lord C, Venter A. Outcome and follow-up studies of high functioning autistic individuals. In Schopler E, Mesibov G, editors. High functioning individuals with autism. New York: Plenum; 1992. p. 187-198.

http://dx.doi.org/10.1007/978-1-4899-2456-8_10

[16] Ozonoff S, Miller JN. Teaching theory of mind: a new approach to social skills training for individuals with autism. $J$ Autism Dev Disord 1995; 25: 415-33. http://dx.doi.org/10.1007/BF02179376

[17] Lezak MD. Neuropsychological assessment. $3^{\text {rd }}$ ed. New York: Oxford University Press; 1995.

[18] Moran S, Gardner H. "Hill, skill, and will:" executive function from a multiple-intelligences perspective. In: Meltzer L, editor Executive function in education: from theory to practice. New York: Guilford Press; 2007. p. 19-38.

[19] Gilotty L, Kenworthy L, Sirian L, Black DO, Wagner AE. Adaptive skills and executive function in autism spectrum disorders. Child Neuropsychol 2002; 31: 241-8. http://dx.doi.org/10.1076/chin.8.4.241.13504

[20] Ozonoff S, Schetter PL. Executive dysfunction in autism spectrum disorders: from research to practice. In: Meltzer L, editor. Executive function in education: from theory to practice. New York: Guilford Press; 2007. p. 133-60.

[21] Jiron C. Brainstorming: using neuropsychology in the schools. Los Angeles: Western Psychological Services; 2004.

[22] Sattler JM, Hoge RD. Assessment of children: behavioral, social, and clinical foundations. $5^{\text {th }}$ ed. La Mesa (CA): Jerome M. Sattler Publisher; 2006.

[23] Miller DC. Essentials of school neuropsychological assessment. Hoboken (NJ): John Wiley \& Sons; 2007.

[24] Baron IS. Neuropsychological evaluation of the child. New York: Oxford University Press; 2004.

[25] Kolb A, Whishaw I. Fundamental of human neuropsychology. $6^{\text {th }}$ ed. New York: Worth; 2009.

[26] Siegel B. Helping children with autism learn: treatment approaches for parents and professionals. New York: Oxford University Press; 2003. 
[27] Minshew NJ, Goldstein G. The pattern of intact and impaired memory functions in autism. J Child Psychol Psychiatry 2001; 42: 1095-101. http://dx.doi.org/10.1111/1469-7610.00808

[28] Baddeley AD. Working memory. Oxford: Clarendon Press; 1986.

[29] Williams DL, Goldstein G, Minshew N. The profile of memory function in children with autism. Neuropsychology 2006; 20 : 21-9. http://dx.doi.org/10.1037/0894-4105.20.1.21

[30] Lord C, Risi S, Pickles A. Trajectory of language development in autistic spectrum disorders. In Rice ML, Warren SF, editors. Developmental language disorders. Mahwah (NJ): Lawrence Erlbaum Associates; 2004. p. 7-29.

[31] Tager-Flusberg $H$, Paul $R$, Lord CE. Language and communication in autism. In: Volkmar F, Paul R, Klin A, Cohen D, editors. Handbook of autism and pervasive developmental disorder. New York: Wiley; 2005. p. 335-64.

[32] Norbury CF, Bishop DVM. Narrative skills in children with communication impairments. Int $\mathrm{J}$ Lang Commun Disord 2003; 38: 287-313. http://dx.doi.org/10.1080/136820310000108133

[33] Tager-Flusberg $\mathrm{H}$. Dissociations in form and function in the acquisition of language in autistic children. In: TagerFlusberg $\mathrm{H}$, editor. Constraints on language acquisition: studies of atypical children. New York: Wiley; 1995. p. 33564.

[34] Diehl J, Bennetto L, Young E. Story recall and narrative coherence of high-functioning children with autism spectrum disorders. J Abnorm Child Psychol 2006; 34: 83-98. http://dx.doi.org/10.1007/s10802-005-9003-x

[35] Baron-Cohen S, Leslie AM, Frith U. Does the autistic child have a "theory of mind?" Cognition 1985; 21: 37-46. http://dx.doi.org/10.1016/0010-0277(85)90022-8

[36] Bruner J, Feldman C. Theories of mind and the problem of autism. In: Baron-Cohen, S, Tager-Flusberg H, Cohen DJ, editors. Understanding other minds: perspectives from autism. Oxford: Oxford University Press; 1993. p. 267-291.

[37] Landa R. Social language use in asperger syndrome and high-functioning autism. In: Klin A, Volkmar FR, Sparrow SS, editors. Asperger syndrome. New York: Guilford; 2000. p. 125-155.

[38] Loveland K, Tunali B. Narrative language in autism and the theory of mind hypothesis: a wider perspective. In: BaronCohen, S, Tager-Flusberg $\mathrm{H}$, Cohen DJ, editors. Understanding other minds: perspectives from autism. Oxford: Oxford University Press; 1993. p. 267-291.

[39] Gillam RB, Pearson NA. Test of narrative language. Austin (TX): Pro-Ed; 2004.

[40] Pence K, Justice LM, Gosse C. Narrative assessment protocol. Columbus $(\mathrm{OH})$ : Preschool Language and Literacy Lab; 2007.

[41] Petersen DB, Spencer TD. The narrative language measures: tools for language screening, progress monitoring and intervention planning. Perspect Lang Learn Educ 2012; 19: 119-29.

http://dx.doi.org/10.1044/lle19.4.119

[42] Miller J, Andriacchi K, Nockerts, A. Assessing language production using salt software: a clinician's guide to language sample analysis. Middleton (WI): SALT Software, LLC; 2011.

[43] Hedberg N, Westby C. Analyzing story-telling skills: theory to practice. Tucson (AZ): Communication Skill Builders; 1993.

[44] McCabe A, Rollins, PR. Assessment of preschool narrative skills. Am J Speech Lang Pathol 1994; 3: 45-55.

[45] Garcia-Winner M. Thinking about you thinking about me. $2^{\text {nd }}$ ed. San Jose (CA): Think Social Publishing; 2007.

[46] Coggins T, Olswang L, Charmichael Olsson H, Timler G. On becoming socially competent communicators: the challenge for children with fetal alcohol exposure. Int Rev Res Ment Retard 2003; 27: 121-50.

http://dx.doi.org/10.1016/S0074-7750(03)27004-X

[47] Social Cognition [homepage on the Internet]. No date [cited 2014 Mar 10] Available from: http://en.wikipedia.org/wiki/ social_cognition

[48] Mundy P, Newell L. Attention, joint attention, and social cognition. Curr Dir Psychol Sci. 2007; 16: 269-74. http://dx.doi.org/10.1111/j.1467-8721.2007.00518.x

[49] Adams C. Practitioner review: assessment of language pragmatics. J Child Psychol Psychiatry 2002; 43: 973-87. http://dx.doi.org/10.1111/1469-7610.00226

[50] Paul R. Language disorders from assessment through adolescence: assessment and intervention. $3^{\text {rd }}$ ed. St. Louis: Mosby Elsevier; 2007.

[51] Prutting C, Kirchner D. A clinical appraisal of the pragmatic aspects of language. J Speech Hear Disord 1987; 52: 10519.

[52] Bishop D. Children's communication checklist. $2^{\text {nd }}$ ed. Boston:Pearson; 2006.

[53] Semel E, Wiig, EH, Secord WA. Clinical evaluation of language fundamentals. $5^{\text {th }}$ ed. Boston:Pearson; 2013

[54] Creaghead N. Strategies for eliciting and targeting pragmatic behavior in young children. Semin Speech Lang 1984; 5 : 241-52.

http://dx.doi.org/10.1055/s-0028-1085181

[55] D'Zurilla TJ, Maydeu-Ollvares A, Kant GL. Age and gender differences in social problem-solving ability. Pers Individ Dif 1998; 25: 241-52. http://dx.doi.org/10.1016/S0191-8869(98)00029-4

[56] Bowers L, Huisingh $R$, LoGiudice C. Social language development test: elementary. East Moline (IL) Linguisystems, Inc; 2008.

[57] Phelps-Terasaki D, Phelps-Gunn T. Test of pragmatic language. $2^{\text {nd }}$ ed. East Moline (IL): Linguisystems, Inc; 2007.

[58] American Speech-Language-Hearing Association. Preferred practice patterns for the profession of speech-language pathology: preferred practice patterns [document on the Internet]. American Speech-Language-Hearing Association; 2004 [cited 2014 Mar 22].Available from: www.asha.org/policy.

[59] Bernthal JE, Bankson NW, Flipsen P. Articulation and phonological disorders: speech sound disorders in children. $6^{\text {th }}$ ed. Boston: Pearson; 2009.

[60] Bauman-Waengler J. Articulatory and phonologica impairments: a clinical focus. $4^{\text {th }}$ ed. Boston: Pearson; 2012.

[61] Gordon-Brannan ME, Weiss CE. Clinical management of articulatory and phonologic disorders. $3^{\text {rd }}$ ed. Philadelphia (PA): Lippincott Williams and Wilkins; 2007

[62] American Speech-Language-Hearing Association. Childhood apraxia of speech: position statement [document on the Internet]. American Speech-Language-Hearing Association; 2007 [cited 2014 Mar 22]. Available from: www.asha.org/policy.

[63] Shriberg LD, Paul R, McSweeny JL, Klin A, Cohen DJ, Volkmar FR. Speech prosody characteristics of adolescents and adults with high-functioning autism and Asperger syndrome. J Speech Lang Hear Res 2001; 44: 1097-115. http://dx.doi.org/10.1044/1092-4388(2001/087)

[64] California Department of Developmental Services. Autism spectrum disorders: best practice guidelines for screening, diagnosis, and assessment [document on the Internet]. California Department of Developmental Services; 2002 [cited $2014 \quad$ Feb 28]. Available from: https://dds.ca.gov/Autism/docs/ASD_Best_Practice2002.pdf.

[65] Kanne SM, Gerber AJ, Quirmbach LM, Sparrow SS, Cicchetti DV, Saulnier CA. The role of adaptive behavior in autism 
spectrum disorders: implications for functional outcome. J Autism Dev Disord 2011; 41: 1007-18. http://dx.doi.org/10.1007/s10803-010-1126-4

[66] Sparrow SS, Cicchetti DV, Balla DA. Vineland adaptive behavior scales. $2^{\text {nd }}$ ed. Minneapolis $(\mathrm{MN})$ : Pearson Assessments; 2005

[67] Achenbach TM, Rescorla LA. Manual for the ASEBA preschool forms and profiles. Burlington (VT): University of Vermont Research Center for Children, Youth, and Families; 2000.

[68] Achenbach TM, Rescorla LA. Manual for the ASEBA preschool forms and profiles. Burlington (VT): University of Vermont Research Center for Children, Youth, and Families; 2001.

[69] de Bildt A, Sytema S, Kraijer D, Sparrow S, Minderaa R. Adaptive functioning and behaviour problems in relation to level of education in children and adolescents with intellectual disability. J Intellect Disabil Res 2005; 49: 672-81. http://dx.doi.org/10.1111/j.1365-2788.2005.00711.x

[70] El-Ghoroury $\mathrm{NH}$, Krackow $\mathrm{E}$. Enhancing the identification of autism spectrum disorders via a model of culturally sensitive childhood assessment. Prof Psychol Res Pr 2013; 43: 24955 http://dx.doi.org/10.1037/a0027354

[71] Ayres J. Sensory integration and the child. Los Angeles: Western Psychological Services; 1979.

[72] Dunn W. The impact of sensory processing abilities on the daily lives of young children and families: a conceptual model. Infants Young Child 1997; 9: 23-35. http://dx.doi.org/10.1097/00001163-199704000-00005

[73] Dunn, W. Supporting children to participate successfully in everyday life by using sensory processing knowledge. Infants Young Child 2007; 20: 84-101. http://dx.doi.org/10.1097/01.IYC.0000264477.05076.5d
$[74]$

Thomchek T, Dunn W. Sensory processing in children with and without autism: a comparative study using the short sensory profile. Am J Occup Ther 2007; 61: 190-200. http://dx.doi.org/10.5014/ajot.61.2.190

[75] Ashburner J, Ziviani J, Rodger S. Sensory processing and classroom emotional, behavioral, and educational outcomes in children with autism spectrum disorder. Am J Occup Ther 2008; 62: 564-73. http://dx.doi.org/10.5014/ajot.62.5.564

[76] Anzalone ME, Williamson GG. Sensory processing and motor performance in autism spectrum disorders. In: Wetherby AM, Prizant BM, editors. Autism spectrum disorders: a transactional developmental perspective. Baltimore (MD): Brookes; 2000. p. 143-66.

[77] Hilton C. Sensory processing and motor issues in autism spectrum disorders. In: Matson JL, Sturmey $\mathrm{P}$, editors. International handbook of autism and pervasive developmental disorders: autism and child psychopathology series. New York: Springer Science + Business Media; 2011. p. $175-93$

[78] Dawson G, Watling R. Interventions to facilitate auditory, visual, and motor integration in autism: a review of the evidence. J Autism Dev Disord 2000; 30: 415-21. http://dx.doi.org/10.1023/A:1005547422749

[79] Dewey D, Cantell M, Crawford SG. Motor and gestura performance in children with autism spectrum disorders developmental coordination disorder, and/or attention deficit hyperactivity disorder. J Int Neuropsychol Soc 2007; 13: 24656.

http://dx.doi.org/10.1017/S1355617707070270

[80] Mattard-Labrecque C, Amor I, Couture M. Children with autism and attention difficulties: a pilot study of the association between sensory, motor, and adaptive behaviors. J Can Acad Child Adolesc Psychiatry 2013; 22: 139-46.

[81] Dyches T. Assessing diverse students with autism spectrum disorders. ASHA Leader 2011; January 18.

\section{DOI: http://dx.doi.org/10.6000/2292-2598.2014.02.01.9}

(c) 2014 Dodd et al.; Licensee Lifescience Global.

This is an open access article licensed under the terms of the Creative Commons Attribution Non-Commercial License (http://creativecommons.org/licenses/by-nc/3.0/) which permits unrestricted, non-commercial use, distribution and reproduction in any medium, provided the work is properly cited. 